\title{
VARIOUS WAVELET TECHNIQUES FOR X-RAYS USING THRESHOLDING
}

\author{
Amandeep Kaur ${ }^{1}$ Dr.Vijay Kumar Joshi ${ }^{2}$
}

Abstract: In recent years, there are many techniques that are used in image processing for de-noising and retaining the edges of an image. There are different noises found in an image and for their removal application dependent de-noising algorithms are used. Each one of them have different pros and cons. The basic idea is to remove the noise and improve the quality of image. In this paper, de-noising techniques are discussed. The aim of denoising techniques is to reduce noise as well to retain the details of image. This paper analyze the performance of the wavelet-based thresholding approach at different level in the existence of Speckle noise and Poison for Wavelet types Daubechies and Mallat for denoising X-ray image by calculating parameters SNR,MSE,EPI,Correlation coefficient.

Keywords: De-noising, Speckle \& Poison Noise, Wavelet.

\section{INTRODUCTION}

The image usually has noise which is not easily eliminated in image processing. According to actual image characteristics, noise statistical property and frequency spectrum distribution rule, people have developed many methods of eliminating noises, which approximately are divided into space and transformation fields The space field is data operation carried on the original image and processes the image grey value, like neighborhood average method, wiener filter, center value filter.

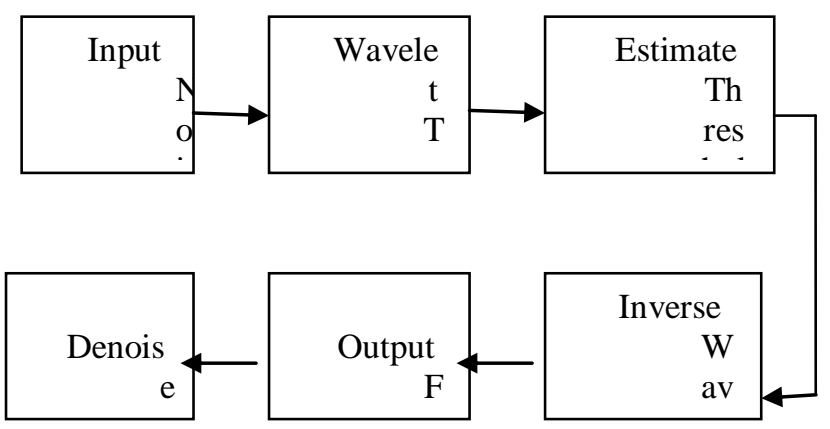

\subsection{TYPES OF NOISES}

Figure 1.1 Block diagram of Image denoising using wavelet transform

The noise is characterized by its pattern and by its probabilistic characteristics. There is a wide variety of noise Types while we focus on the most important types, which are; Gaussian noise, salt and pepper noise, poison noise, impulse noise, speckle noise.

- Poison Noise: Poisson noise is induced by the nonlinear response of the image detectors and recorders. This type of noise is image data dependent. This term arises because detection and recording processes involve random electron emission having a Poisson distribution with a mean response value. Since the mean and variance of a Poisson distribution are equal, the image dependent term has a standard deviation if it is assumed that the noise has a unity variance.

- Speckle Noise: Speckle is a complex phenomenon, which degrades image quality with a backscattered wave appearance which originates from many microscopic diffused reflections that passing through internal organs and makes it more difficult for the observer to discriminate fine detail of the images in diagnostic examinations.

\section{Wavelet Transform}

\footnotetext{
${ }^{1}$ Student Masters of Technology, Department of Computer Science and Engineering, LCET, Katani Kalan,Punjab,India

${ }^{2}$ Professor and Head of Department, Department of Computer Science and Engineering, LCET, Katani Kalan,Punjab,India
} 
The wavelet expansion set is not unique. A wavelet system is a set of building blocks to construct or represents a signal or function. It is a two dimensional expansion set, usually a basis, for some class one or higher dimensional signals. The wavelet expansion gives a time frequency localization of the signal. Wavelet systems are generated from single scaling function by scaling and translation. A set of scaling function in terms of integer translates of the basic scaling function by

$\varphi k^{(t)}=\varphi(t-k) \mathrm{K} \in \mathrm{z} \varphi € L^{2} \ldots \ldots$ (i)

\section{Daubechies Wavelet Transform}

The Daubechies Wavelets are compactly supported and have highest number of vanishing moments. The types are db1, $\mathrm{db} 2 \ldots \mathrm{db} 45$. Discrete Wavelet Transform and continuous wavelet transform is possible for these. These are not symmetrical. The length of the filter is $2 \mathrm{~N}$. The number of vanishing moments are $\mathrm{N}$. This wavelet use overlapping window so the high frequency coefficient spectrum display all frequency changes. So this is useful in compression and for the removal of noise in audio signal processing.

\section{Mallat Wavelet Transform}

It computes DWT wavelet coefficients for a finite set of input

data, which is a power of 2 . This input data is passed through two convolution functions, each of which creates an output stream that is half the length of the original input. This method is referred to as down sampling. The convolution functions are filters. One half of the output is come through the low pass filter function and the other half is generated by the high pass filter function. The low pass outputs carry most of the information of the input signal and are known as "coarse" coefficients. The outputs from the high pass filter are called as "detail" coefficients. The parameters obtained from the low pass filter are used as the original signal for the next set of coefficients. This process is carried out repeatedly until a trivial number of low pass filter coefficients are left. The final output hold the remaining low pass filter outputs and the accumulated high pass filter outputs. This strategy is called as decomposition.

In some applications, some form of processing is done to the wavelet coefficients obtained after the DWT. Once the processing is completed, the data vector is built back from the coefficients. This process of reconstruction is referred to as the inverse Mallat's algorithm.

In the reconstruction procedure, quadrature mirror filters Equation are supplied with the coarse coefficients and the accumulated detail coefficients. The outputs from the two filters are added and are considered as the coarse coefficients for the next stage of reconstruction. This process is continued until the data vector is obtained.

\section{THRESHOLDING METHODS}

Thresholding is a simple non-linear technique, which operates on one wavelet coefficient at a time. In its most basic form, each coefficient is thresholded by comparing against threshold, if the coefficient is smaller than threshold, set to zero; otherwise it is kept or modified. Replacing the small noisy coefficients by zero and inverse wavelet transform on the result may lead to reconstruction with the essential signal characteristics and with less noise. It is important to know about the two categories of thresholding.

- Hard Thresholding: In hard thresholding all coefficients whose magnitude is greater than the selected threshold value $\lambda$ remains same and the others whose magnitude is smaller than $\lambda$ are set to zero. It creates a region around zero where the coefficients are considered negligible.

- Soft Thresholding: In soft thresholding, the coefficients whose magnitude is greater than the selected threshold value are shrunk towards zero by an amount of threshold $\lambda$ and others set to zero.

\section{METHODOLOGY}

Methodology begins with the study of wavelet. Then various types of noise have been studied and denoising methods. So methodology to fulfill objective is described as follow:

- Techniques (Mallat and Daubechies) for denoising can be used.

- Denoising of the Images using some parameters and then find out the thresholding.

- Compare the performance of proposed method with existing approaches.

The operation for calculating the effectiveness is as follow:

$>$ Add noise to the Image

$>$ Choose Wavelet type

$>$ Select decomposition level

$>$ Implement thresholding approach

$>$ Perform the denoising of image

$>$ Calculate SNR,MSE,EPI,CC

\section{PERFORMANCE EVALUATION}


In order to find out the performance of wavelet the results are calculated of four parameters like Signal to noise ratio(SNR),Mean square error(MSE),Edge preservation Index(EPI),Correlation Coefficient(CC). From results it is clear that wavelet thresholding gives best results.

\section{RESULTS}

In order to check the performance of various wavelets Speckle and Poison noise is added artificially into X-Ray test image objects.

\begin{tabular}{|l|l|l|l|l|}
\hline & Sing HT & Sing ST & Mallat using HT & Mallat using ST \\
\hline & 42 & 78 & 22 & 34 \\
\hline & 56 & 32 & 72 & 69 \\
\hline 60 & 35 & 60 & 38 \\
\hline
\end{tabular}

Tabale-I Comparison table of SNR of Speckle Denoised Image at different Noise levels

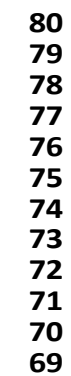

Comparison Chart of SNR of Speckle Denoised Image at different Noise levels

\begin{tabular}{|l|l|l|l|l|}
\hline & Sing HT & sing ST & Mallat Using HT & Mallat using ST \\
\hline & 99 & 20 & 33 & 70 \\
\hline & 82 & 71 & 56 & 69 \\
\hline 47 & 34 & 29 & 36 \\
\hline
\end{tabular}

Table-II Comparison table of SNR of Poison Denoised Image at different Noise levels
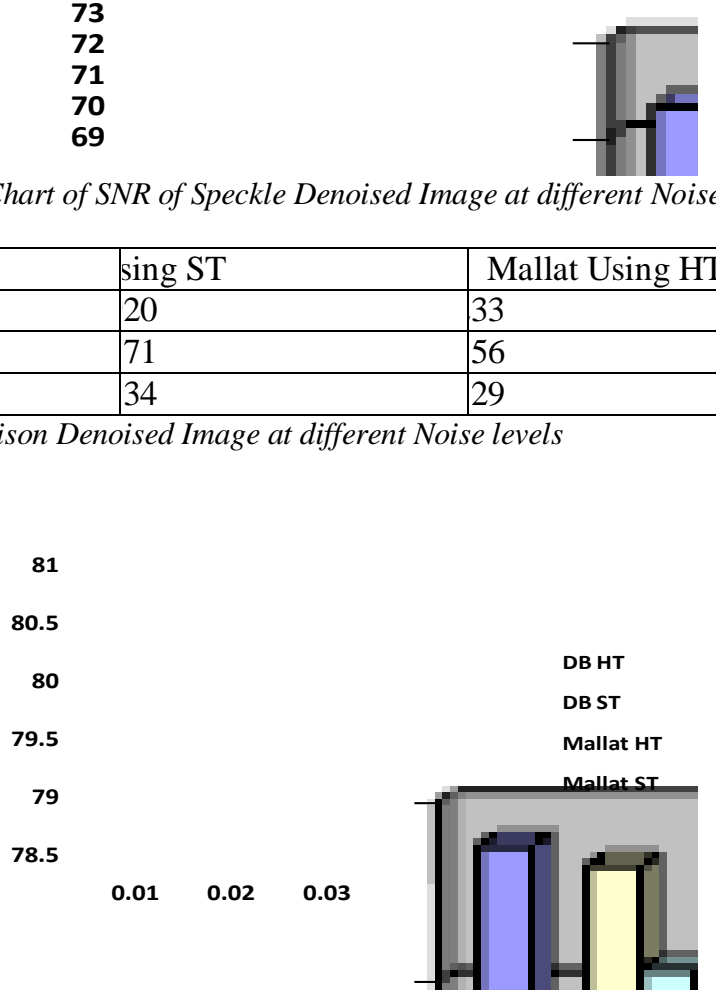

Comparison Chart of SNR of Poison Denoised Image at different Noise levels

\begin{tabular}{|l|l|l|l|l|}
\hline & sing HT & sing ST & t using HT & t using ST \\
\hline & & & & \\
\hline & & & & \\
\hline & & & & \\
\hline
\end{tabular}

Table-III Comparison table of MSE of Speckle Denoised Image at different Noise levels 


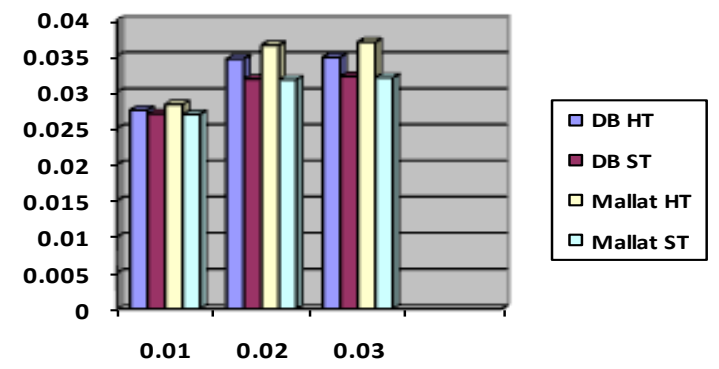

Comparison Chart of MSE of Speckle Denoised Image at different Noise levels

\begin{tabular}{|l|l|l|l|l|}
\hline & Sing HT & Sing ST & t Using HT & t using ST \\
\hline & & & & \\
\hline & & & & \\
\hline & & & & \\
\hline
\end{tabular}

Table-IVComparison table of MSE of Poison Denoised Image at different Noise levels.

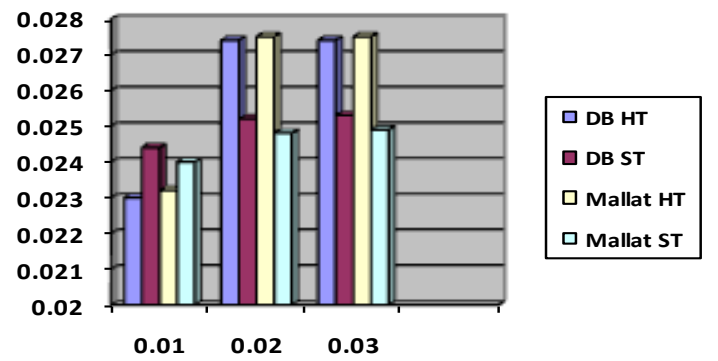

Comparison chart of MSE of Poison Denoised Image at different Noise levels.

\begin{tabular}{|l|l|l|l|l|}
\hline Noise & DB using HT & DB using ST & Mallat using HT & Mallat using ST \\
\hline .01 & .1309 & .1286 & .1304 & .1316 \\
\hline .02 & .1291 & .1280 & .1291 & .1297 \\
\hline .03 & .1269 & .1279 & .1263 & .1279 \\
\hline
\end{tabular}

Table-V Comparison table of EPI of Speckle Denoised Image at different Noise level

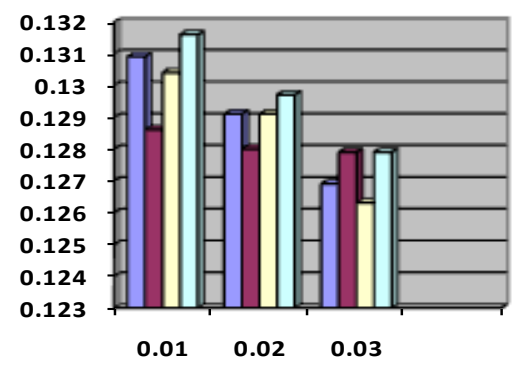

\begin{tabular}{|l|}
\hline$\square$ DB HT \\
$\square$ DB ST \\
$\square$ Mallat HT \\
$\square$ Mallat ST
\end{tabular}

Comparison Chart of EPI of Speckle Denoised Image at different Noise level

\begin{tabular}{|l|l|l|l|l|}
\hline Noise & DB using HT & DB using ST & Mallat using HT & Mallat using ST \\
\hline .01 & .1341 & .1300 & .1343 & .1307 \\
\hline .02 & .1340 & .1298 & .1338 & .1299 \\
\hline
\end{tabular}




\begin{tabular}{|l|l|l|l|l|}
\hline .03 & .1336 & .1299 & .1334 & .1297 \\
\hline
\end{tabular}

Table-VI Comparison table of EPI of Poison Denoised Image at different Noise level

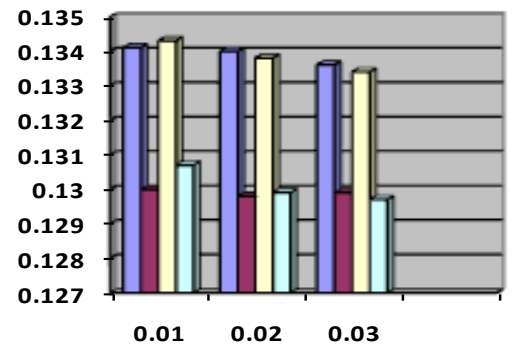

\begin{tabular}{|l|}
\hline$\square$ DB HT \\
$\square$ DB ST \\
$\square$ Mallat HT \\
$\square$ Mallat ST \\
\hline
\end{tabular}

Comparison Chart of EPI of Poison Denoised Image at different Noise level

\begin{tabular}{|l|l|l|l|l|}
\hline Noise & DB using HT & DB using ST & Mallat using HT & Mallat using ST \\
\hline .01 & .9959 & .9961 & .9957 & .9959 \\
\hline .02 & .9884 & .9902 & .9881 & .9903 \\
\hline .03 & .9834 & .9860 & .9829 & .9863 \\
\hline
\end{tabular}

Table-VII Comparison table of CC of Speckle Denoised Image at different Noise levels

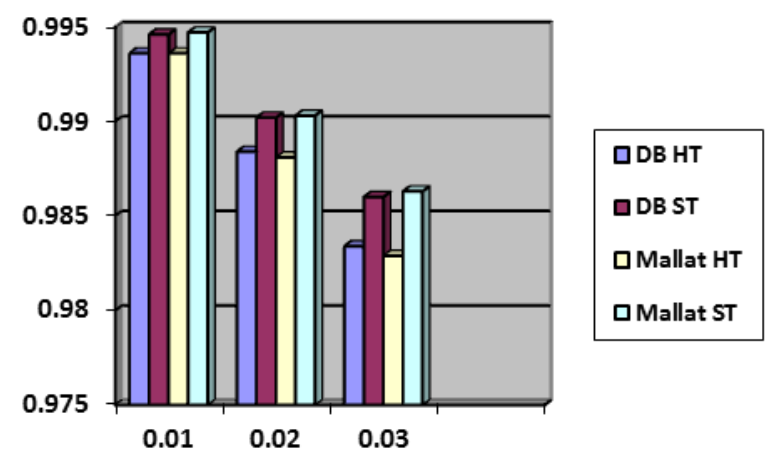

Comparison Chart of CC of Speckle Denoised Image at different Noise levels

\begin{tabular}{|l|l|l|l|l|}
\hline Noise & DB using HT & DB using ST & Mallat using HT & Mallat using ST \\
\hline .01 & .9971 & .9968 & .9971 & .9969 \\
\hline .02 & .9960 & .9966 & .9959 & .9967 \\
\hline .03 & .9960 & .9966 & .9959 & .9967 \\
\hline
\end{tabular}

Table-VIII Comparison table of CC of Poison Denoised Image at different Noise level
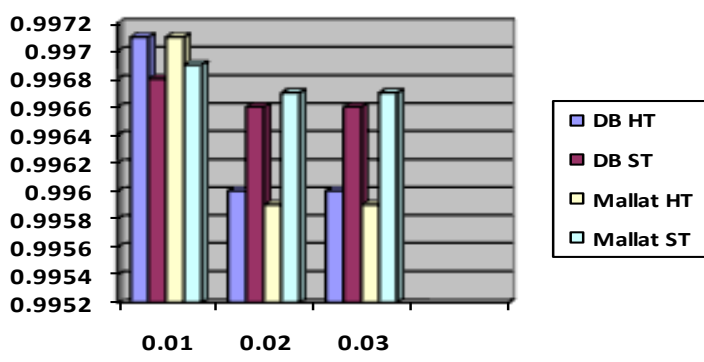

Comparison Chart of CC of Poison Denoised Image at different Noise levels 


\section{CONCLUSION \& FUTURE SCOPE}

In this paper, denoising of medical X-Ray images is performed using Daubechies and Mallat wavelet by using hard and soft thresholding techniques. Results shows that Daubechies wavelet gives best results for hars thersholding and Mallat for soft. The parameters are calculated inorder to remove Speckle and Poison noise of the images .In future it can be used for images like CT-Scan and Ultrasound for different types of noises.

\section{REFERENCES}

[1] R.A.Devore,B.J.Lucier(1992),'”Fast Wavelet techniques for Near-optimal Image processing,IEEE press, New York ”,pp. 1129-1135.

[2] Donoho.D.L, Johnstone.I.M (1994),' ideal spatial adaptation via wavelet shrinkage .Biometrika, 81, pp.425-455.

[3] Robert D.Nowak(1999),’Wavelet Based Rician Noise Removal “,IEEE Transactions on image processing,vol.8,no.10,pp.1408,October 1999.

[4] D.S and F.J (2001),"Artifact Free signal Denoising With Wavelets", International Conference on Acoustics, Speech and Signal Processing.

[5] B.S and P.S (2004),"Filtered Wavelet thresholding methods", Journal of Computational and Applied Mathematics.

[6] M.S.K,P.Dr.S and S.Dr.M(2008),'Image Denoising using Discrete Wavelet Transform”, International Journal of Computer Science and Network Security Vol.8 No.1,January 2008.

[7] Kaur.A and Singh.K (2010),'Speckle Noise Reduction by Using Wavelets", National conference on Computational Instrumentation, Csio Chandigarh, India, 19-20 March.

[8] Hassan.H and Saparon.A (2011),'Still Image Denoised Based on Discrete Wavelet Transform”, IEEE International Conference on System Engineering and Technology, pp 188-192.

[9] Shinde.B, Mhaske.D, Patare.M and Dani.A.R(2012),” Apply Different Filtering Techniques To Remove The Speckle Noise Using Medical Images”, International Journal Of Engineering Research And Applications (IJERA) Issn: 2248-9622, Vol. 2, Issue 1, Jan-Feb 2012.

[10] Agrawal.S and Sahu.R (2012), 'Wavelet Based MRI Image Denoising Using Thresholding Techniques”, International Journal of Science, Engine ering and Technology Research, Volume 1, Issue 3, pp 32-35.

[11] Sarage.G.N and Jambhorkar.Dr.S (2012), ’Enhancement of Chest X-ray Images Using Filtering Techniques",International Journal of Advanced Research in Computer Science and Software Engineering, Volume 2,Issue 5.

[12] Sidhu.K.S.,Khaira.B.S and Virk.I.S (2012)" Medical Image Denoising In the Wavelet Domain Using Haar And DB3 Filtering ",International Refereed Journal of Engineering and Science, Volume 1,pp 1-8.

[13] Vishwa.A and Shilpa (2012),"Speckle Noise Reduction in Ultrasound Images by Wavelet Thresholding", International Journal of Advanced Research In Computer Science and Software Engineering, Volume 2.

[14] Bahendwar.Y and Sinha.Dr.G.R (2012)," A Modified Algorithm For Denoising Mri Images Of Lungs Using Discrete Wavelet Transform", National Conference on Innovative Paradigms in Engineering \& Technology (NCIPET-2012) Proceedings published by International Journal of Computer Applications $®$ (IJCA).

[15] Dewangan.N and Goswami.A.D (2012),'Image Denoising Using Wavelet Thresholding Methods", International Journal of Engineering Sciences \& Management, Vol.2, Issue 2, pp.271-275.

[16] Subbuthai.P, Kavithabharathi.K and Muruganand.S (2013),'Reduction of types of Noises in dental images", International Journal of Computer Applications Technology and Research, Volume 2, Issue 4.

[17] Kaur.S, Kaur.G and Singh.Dr.D (2013), "Comparative Analysis of Haar and Coiflet Wavelets using Discrete Wavelet Transform in Digital Image Compression, International Journal of Engineering Research and Applications, Vol.3,Issue 3,pp.669-673.

[18] Kaur.R and Kaur.R (2013), "Survey of De-noising Methods Using Filters and Fast Wavelet Transform", International Journal of Advanced Research in Computer Science and Software Engineering, Volume 3, Issue 2, pp.133-136.

[19] Kaur.R and Kaur.R (2013),'Image Denoising Based on Wavelet Techniques Using Thresholding for Medical Images", International Journal of Computer Trends and Technology, Volume 4, Issue 8, pp.2526-2533.

[20] Kumar.H and Roy.D.K (2014),"A Novel Color Image Denoising Technique Using Window Based Soft Fuzzy Filter", International Journal of Advanced Research in Computer Engineering \& Technology, Volume 3, Issue 8.

[21] Soni.V.K and Karanjgaokar.V (2014),’Wavelet Based Noise Reduction in Medical Image”, International Journal on Advanced Computer theory and Engineering, Volume 3, Issue 3.

[22] S.S and S.G.P (2015),"Comparative Analysis of Various Wavelets for Denoising Color Images", ARPN Journal of Engineering and Applied Sciences, Vol.10, No.9.

[23] Singh.S and Wadhwani.S (2015),"Medical Image Denoising Using Sub Band Adaptive Thresholding Techniques Based on Wavelet 2D Transform",International Journal of Bio-Science and Bio-Technology, Vol.7.

[24] Kumar.P and Agarwal.S.K (2015),"Analysis of Wavelet Denoising of a Colour Image with Different Types of Noises", International Journal of Signal Processing, Image Processing and Pattern Recognition, Vol.8, pp.125-134.

[25] Rahman.M.A and Khan.A.S (2016),"A Spatial Spectral Filtration (SSF) Based Correlated Coefficients Thresholding Approach for Image Denoising”, International Journal of Computer Science and Information Technologies, Vol.7, pp 1097-1101.s 